\title{
Polimorfismos de p53 y enfermedad coronaria.
}

\author{
Hugo Verdejo
}

Recibido el 13 de julio de 2009. Aceptado el 18 de julio de 2009

Rev Chil Cardiol 2009; 28: 202-204

La proteína supresora de tumores p53 (TP53) es una molécula esencial en el control del ciclo celular y de la muerte celular programada (apoptosis), por lo cual gran parte de la investigación asociada se ha centrado en su participación en el proceso de carcinogénesis. La asociación con p53 y ateroesclerosis fue recién reportada hacia fines de los '90 por Guevara et al, quienes demostraron que la inactivación de p53 en un modelo animal de ateromatosis espontánea (Ratones apoE knockout) se asociaba a una progresión acelerada de las placas ateroescleróticas en presencia de niveles de colesterol sanguíneo similares ${ }^{1}$. Algunos años después, van Vlijmen et al 2 corroboraron estos hallazgos trasplantando ratones apoE*3-Leiden (una variante de los ratones ApoE knockout) con medula ósea proveniente de ratones p53 knockout. Al igual que el grupo anterior, las lesiones ateroscleróticas del grupo de ratones trasplantados eran significativamente mayores, alcanzando un tamaño promedio de 2.3 veces las presentes en los ratones control. Ambos estudios in vivo parecen sostener la importante conclusión de que un déficit de actividad de p53 en la población de monocitos/macrófagos de la placa se asocia a un mayor tamaño de lesión aterosclerótica 3; sin embargo, la relación causal que liga ambas observaciones sigue siendo elusiva.

Es bien sabido que condiciones de stress celular, como el causado por hipoxia o exposición a lipoproteínas oxidadas activa p53 mediante la inhibición de su degradación ${ }^{4}$. Aún cuando las vías de transducción precisas mediante las cuales la actividad de p53 modula la progresión de la aterosclerosis no son conocidas, existen al menos 3 mecanismos alternativos que permiten explicar cómo un incremento en la muerte por apoptosis de los macrófagos de la placa promueve la progresión de la enfermedad:

1. La muerte por apoptosis de los macrófagos puede asociarse a liberación de contenidos celulares tóxicos desde las células moribundas 5 .

2. La fagocitosis de los cuerpos apoptóticos puede ser inhibida en un ambiente rico en lipoproteínas oxidadas 6 , promoviendo la acumulación de lípidos modificados en la placa y la activación de las cascadas inflamatorias

3. Finalmente, aún cuando los macrófagos residentes logren fagocitar estos remanentes ricos en lípidos, la acumulación de excesos de colesterol no esterificado constituye un estrés citotóxico que promueve la muerte celular y la liberación de moléculas proinflamatorias ${ }^{7}$.

La descripción de polimorfismos en el gen TP53 ha reactivado el interés por la probable participación de esta proteína en la enfermedad cardiovascular. El polimorfismo investigado por los autores Caamaño et $a^{1} 8$ en este número de la Revista Chilena de Cardiología (Polimorfismo Pro72Arg) es particularmente interesante dada la existencia de diferencias biológicas en la actividad de la proteína; así, la forma TP53 P72 (Pro) se asocia a un rol predominantemente antiproliferativo, promoviendo el secuestro de las células en G1; por el contrario, la variante TP35 R72 (Arg) se asocia más bien a un efecto pro-apoptótico9,10 y es más susceptible a degradación inducida por la E6 oncoproteína del papilomavirus humano ${ }^{11}$, lo que ha sido propuesto como mecanismo subyacente a la asociación entre el polimorfismo R72 y el cáncer cervicouterino.

Correspondencia: Dr. Hugo Verdejo P.

Departamento de Enfermedades Cardiovasculares

Pontificia Universidad Católica de Chile

Correo Electrónico:heverdej@uc.cl 
En el primer reporte en humanos vinculando polimorfismos de TP53 y enfermedad cardiovascular, Kojima et al12 incluyeron 66 pacientes consecutivos sometidos a angioplastía y seguidos por 3 meses. En los pacientes homocigotos para la forma TP53 R72 (Arg) se observó una mayor tasa de reestenosis a 3 meses (diámetro luminal mínimo $0.95 \pm 0.63$ vs. $1.24 \pm 0.81$ para homocigotos TP53 P72, $\mathrm{p}<0.01$ ). Estos hallazgos fueron confirmados por Zee et al13, quienes incluyeron 779 pacientes sometidos a angioplastía exitosa, definiendo como casos a aquellos pacientes con estenosis mayor al $50 \%$ en el vaso tratado al momento del seguimiento (6 meses). Los autores evaluaron 3 polimorfismos del gen TP53 (11951_11966dup16bp, 13494G>A y R72P) demostrando que dos haplotipos del gen TP53, 16bp--P72-G13494, y 16bp \pm P72-A13494 tenían un efecto protector en la tasa de reestenosis luego del ajuste por índice de masa corporal, historia de hipertensión, dislipidemia, tabaquismo, diabetes, infarto, tipo de lesión e historia familiar de enfermedad coronaria. De paso, los autores evidencian la compleja relación entre las variantes alélicas de un determinado gen y las limitaciones inherentes de los estudios centrados en un único polimorfismo en establecer patrones de predicción exactos y reproducibles.

En esta edición de la Revista Chilena de Cardiología, los autores Caamaño et al. reportan la asociación entre el polimorfismo Pro72Arg del gen TP53 y la presencia de enfermedad coronaria en la población chilena. Para tal efecto, se evaluó la presencia del polimorfismo en cuestión en 209 pacientes con enfermedad coronaria conocida definida por coronariografía y en 216 individuos controles identificados mediante un cuestionario, demostrando una diferencia significativa en la prevalencia del polimorfismo en la población con cardiopatía coronaria. Los resultados de los autores son consistentes con las prevalencias anteriormente reportadas para el polimorfismo $\mathrm{P} 72 \mathrm{R}$ en población adulta nacional ${ }^{14}$. Sin embargo, sus resultados con respecto al efecto del polimorfismo en la incidencia de enfermedad coronaria no han sido reproducidos por ningún otro estudio poblacional independientemente del criterio empleado para definir enfermedad cardiovascular (por anamnesis o mediante coronariografía) ${ }^{15-17}$. En consideración a lo anterior, es necesario interpretar con cautela estos hallazgos, considerando las limitaciones metodológicas del trabajo, en particular la definición del grupo control y la significativa diferencia de edad entre el grupo de pacientes coronarios vs. el grupo control ( $62 \pm 10$ vs. $42 \pm 8$ años, $p<0.001$ ); futuros estudios poblacionales basados en metodologías que permiten el análisis simultáneo de gran número de genes junto a una definición rigurosa de los casos y controles permitirán esclarecer el rol de los polimorfismos de p53 como factores pronósticos en el desarrollo de enfermedad coronaria.

\section{Referencias}

1. GUEVARA NV, KIM HS, ANTONOVA EI, CHAN L. The absence of p53 accelerates atherosclerosis by increasing cell proliferation in vivo. Nat Med 1999; 5: 335-9.

2. VAN VLIJMEN BJ, GERRITSEN G, FRANKEN AL, BOESTEN LS, KOCKX MM, GIJBELS MJ, et al. Macrophage p53 deficiency leads to enhanced atherosclerosis in $\mathrm{APOE}^{\star} 3$-Leiden transgenic mice. Circ Res 2001; 88: 780-6.

3. TABAS I. p53 and atherosclerosis. Circ Res 2001; 88: 747-9.

4. LOHRUM MA, VOUSDEN KH. Regulation and function of the p53-related proteins: same family, different rules. Trends Cell Biol 2000; 10: 197-202.

5. DARZYNKIEWICZZ, BEDNER E, TRAGANOS F, MURAKAMI $T$. Critical aspects in the analysis of apoptosis and necrosis. Hum Cell 1998; 11: 3-12.

6. CHANG MK, BERGMARK C, LAURILA A, HÖRKKÖ S, HAN KH, FRIEDMAN $P$, et al. Monoclonal antibodies against oxidized low-density lipoprotein bind to apoptotic cells and inhibit their phagocytosis by elicited macrophages: evidence that oxidation-specific epitopes mediate macrophage recognition. Proc Natl Acad Sci U S A 1999; 96: 6353-8.

7. TABAS I. Atherosclerosis: cell biology and lipoproteins. Curr Opin Lipidol 1997; 8: U25-U27.

8. CAAMAÑO J, SAAVEDRA N, JARAMILLO P, LANAS C, LANAS F, SALAZAR L. Polimorfismo Pro72Arg del gen TP53 se asocia a enfermedad coronaria en individuos chilenos. Rev Chil Cardiol 2009; 28: 151-157

9. DUMONT P, LEU JI, DELLA PIETRA AC, GEORGE DL, MURPHY M. The codon 72 polymorphic variants of $p 53$ have markedly different apoptotic potential. Nat Genet 2003; 33: 3 57-65.

10. PIM D, BANKS L. p53 polymorphic variants at codon 72 exert different effects on cell cycle progression. Int $\mathrm{J}$ Cancer 2004; 108: 196-9. 
11. STOREY A, THOMAS M, KALITAA, HARWOOD C, GARDIOL $D$, MANTOVANI F, et al. Role of a p53 polymorphism in the development of human papillomavirus-associated cancer. Nature 1998; 393: 229-34.

12. KOJIMA S, GOTO Y, NONOGI H, HORIE H, KINOSHITA M, IWAI N. Role of a p53 polymorphism in luminal narrowing after balloon coronary angioplasty. Atherosclerosis 2000; 151 : 585-6.

13. ZEE RY, COOK NR, KIM CA, FERNANDEZ-CRUZ A, LINDPAINTNER K. TP53 haplotype-based analysis and incidence of post-angioplasty restenosis. Hum Genet 2004; 114: 386-90.

14. OJEDA JM, AMPUERO S, ROJAS P, PRADO R, ALLENDE JE, BARTON SA, et al. p53 codon 72 polymorphism and risk of cervical cancer. Biol Res 2003; 36: 279-83.

15. ALKHALAF M, AL-BUSTAN S, HAMODA H, ABDELLA N. Polymorphism of p53 gene codon 72 in Kuwaiti with coronary artery disease and diabetes. Int J Cardiol 2007; 115: 1-6.

16. MANFREDI S, MASETTI S, BOTTO N, COLOMBO MG, TERRAZZI M, VASSALLE C, et al. P53 codon 72 polymorphism in coronary artery disease: no evidence for association with increased risk or micronucleus frequency. Environ Mol Mutagen 2002; 40: 110-5.

17. SMITH MA, SILVA MD, CENDOROGLO MS, RAMOS LR, ARAUJO LM, LABIO RW, et al. TP53 codon 72 polymorphism as a risk factor for cardiovascular disease in a Brazilian population. Braz J Med Biol Res 2007; 40: 1465-72. 